\title{
Registres de santé - recommandations communes
}

\author{
Petra Buscha ${ }^{a}$ Regula Heller ${ }^{a}$, Angelina Hofstetter ${ }^{b}$, Stefanie Hostettler ${ }^{c}$, Esther Kraft ${ }^{\mathrm{c}}$, Agnes Nienhaus ${ }^{\mathrm{d}}$, \\ Michelle Salathée \\ ${ }^{a} \mathrm{ANQ} ;{ }^{b} \mathrm{H}+;{ }^{c} \mathrm{FMH} ;{ }^{d}$ unimedsuisse; ${ }^{\text {e }} \mathrm{ASSM}$; les noms des auteures figurent dans l'ordre alphabétique.
}

Les registres offrant des données fiables revêtent toujours plus d'importance dans le milieu de la santé et leur nombre ne cesse de croître avec l'augmentation du volume de données. Soucieuses de garantir leur qualité, les organisations ANQ, ASSM, FMH, $\mathrm{H}+$ et unimedsuisse ont publié des recommandations communes qui contiennent des standards minimaux en matière de protection et de qualité des données.

Les patients, les fournisseurs de soins et les politiciens intervenant dans le domaine de la santé ont un intérêt légitime à ce que le système de santé soit de qualité élevée tout en restant financièrement supportable. A cet égard, les registres médicaux occupent une fonction essentielle: partie intégrante de l'assurance-qualité, ils contribuent à la transparence et à la comparabilité des prestations médicales, sont à la base de la recherche clinique et épidémiologique et fournissent des données importantes pour la politique de santé et la planification des soins. Il existe un grand nombre de registres (http://www.fmh.ch/fr/asqm/_service/plateforme suisse_des_registre.cfm), aux tâches et objectifs différents selon le champ d'application (et les bases légales). Dans les recommandations présentées ici, on entend par «registre de santé» le relevé systématique de données de santé personnelles (collectées sur la base de critères définis).

\section{Destinataires et objectifs des recommandations}

Ces recommandations s'adressent en premier lieu aux responsables de registres, à leurs collaborateurs et aux participants, et en deuxième lieu, aux organes dirigeants d'hôpitaux et d'autres fournisseurs de soins, aux assureurs-maladie et accident, ainsi qu'aux institutions politiques impliquées dans la planification des soins. Elles fournissent des informations permettant de vérifier la qualité des registres médicaux avant et/ou après leur création, et de garantir que

- les registres médicaux disposent d'une structure adéquate pour mener à bien leur mandat;

- les droits de la personnalité des personnes dont les données de santé sont collectées sont protégés;
- les organisations en charge de registres puissent s'en inspirer;

- les responsables de registres et leurs collaborateurs disposent des compétences spécialisées et techniques requises;

- les ressources humaines et financières nécessaires sont à disposition;

- le relevé, la saisie et l'utilisation des données ont lieu selon des critères clairement définis;

- la qualité des données est assurée et attestée;

- l'utilisation des données pour la recherche et l'assurance-qualité est réglementée.

Les recommandations ne sont pas juridiquement contraignantes, mais bénéficient d'un large soutien des principaux acteurs de la santé grâce à une consultation étendue et à une acceptation élevée. Elles seront évaluées périodiquement, et le cas échéant adaptées, par les organisations en charge de la publication ANQ, FMH, H+, ASSM et unimedsuisse.

\section{Recommandations concernant les registres de santé}

Les Recommandations concernant la création et la gestion de registres dans le domaine de la santé sont disponibles sous la forme d'un flipbook et d'un fichier PDF sur le site www.asqm.ch à la rubrique Publications $\rightarrow$ Recommandations registres.

Vous trouverez ci-après la liste de contrôle pour la vérification des registres de santé.

Pour toute question ou demande de renseignement, les organisations FMH, ANQ, H+, ASSM et unimedsuisse se tiennent à votre entière disposition (ddq[at]fmh.ch; 03135911 11). 


\section{Liste de contrôle}

\section{Planification d'un registre: clarifications préalables}

1.1 La nécessité/l'utilité du registre est décrite pour toutes les personnes impliquées y compris les patients.

1.2 Les conditions-cadres juridiques sont clarifiées.

1.3 L'intégration du registre est clarifiée.

1.4 Le contexte et les éventuels liens d'intérêts sont présentés de manière transparente.

1.5 La conception et le financement à long terme sont assurés, un concept de financement a été établi.

\section{Conception du registre}

2.1 Le but et les tâches du registre sont clairement définis et les questions auxquelles celui-ci doit répondre sont formulées.

2.2 L'organisation du registre est clairement présentée dans un concept/règlement.

\section{Compétences relatives à la gestion du registre}

3.1 Les compétences des gestionnaires du registre correspondent aux objectifs.

3.2 Les compétences spécialisées sont assurées (compétences méthodologiques, expérience clinique dans le domaine concerné).

3.3 Les compétences techniques sont garanties (conception du registre, processus, logistique, qualité et sécurité de la banque de données).

\section{Protection et souveraineté des données}

4.1 Un règlement des données a été établi pour régler les aspects suivants:

a) Protection de la personnalité: description des procédures d'anonymisation et de cryptage des données, information aux patients et consentement ainsi que droit de consulter les données, marche à suivre en cas de rétractation et conservation des données.

b) Accès aux données, souveraineté des données, droit de consulter et droits d'accès, utilisation des données par des tiers. 


\section{Collecte et utilisation des données}

5.1 Les variables à collecter sont clairement définies et correspondent aux objectifs.

5.2 Les structures techniques sont adéquates et évolutives.

5.3 Le rattachement à des données administratives/officielles, à savoir la possibilité d'intégration dans les systèmes informatiques des cliniques (interopérabilité), est garanti.

5.4 Un diagramme de flux de données a été établi et décrit de manière comprëhensible le relevé, la transmission et le traitement des données.

5.5 Un concept d'évaluation a été établi et décrit précisément l'analyse des données et la présentation des résultats.

5.6 Un concept de publication a été établi et décrit précisément les exigences, le contenu et la forme de la publication ainsi que les groupes cibles.

5.7 La réutilisation des données à des fins de recherche est encouragée.

\section{Assurance-qualité}

6.1 Un concept de validation y c. procédure d'examen périodique a été établi et garantit le maintien de la qualité des données.

6.2 L'adéquation des objectifs et des tâches du registre est évaluée périodiquement.

\section{Changement d'objectif et suppression du registre}

7.1 La procédure en cas de changement d'objectif est réglée.

7.2 La procédure en cas de suppression du registre est réglée. 